\title{
Multidrug-resistant enterobacteria colonize commercial day-old broiler chicks in Nigeria
}

\author{
Obianuju Nkiruka Okorafor ${ }^{1}$, Madubuike Umunna Anyanwu² ${ }^{2}$ Emmanuel Onyeka Nwafor ${ }^{1}$, George Nnamdi Anosa ${ }^{1}$ and \\ Rita Ijeoma Udegbunam ${ }^{3}$
}

\begin{abstract}
1. Department of Veterinary Medicine, University of Nigeria, Nsukka, Nigeria; 2. Department of Veterinary Pathology and Microbiology, University of Nigeria, Nsukka, Nigeria; 3. Department of Veterinary Surgery, University of Nigeria, Nsukka, Nigeria.

Corresponding author: Madubuike Umunna Anyanwu, e-mail: madubuike.anyanwu@unn.edu.ng Co-authors: ONO: obianuju.okoroafor@unn.edu.ng, EON: nwaforemmanuel42@gmail.com, GNA: george.anosa@unn.edu.ng, RIU: rita.udegbunam@unn.edu.ng

Received: 01-10-2018, Accepted: 08-02-2019, Published online: 16-03-2019
\end{abstract}

doi: 10.14202/vetworld.2019.418-423 How to cite this article: Okorafor ON, Anyanwu MU, Nwafor EO, Anosa GN, Udegbunam RI (2019) Multidrug-resistant enterobacteria colonize commercial day-old broiler chicks in Nigeria, Veterinary World, 12(3): 418-423.

\begin{abstract}
Aim: This study was conducted to isolate generic enterobacteria from day-old broiler chicks in Nigeria, determine the antibacterial resistance profile, and assess multidrug resistance.

Materials and Methods: The birds were sourced from five purposively-selected hatcheries (identified as A, B, C, D and E) in Southwest Nigeria. Non-duplicate cloacal swabs were collected from a total of 75 (15 birds per hatchery) randomly selected apparently healthy birds. Sampling was done in three batches of five chicks per batch at 2-week interval. Isolation of enterobacteria was done using MacConkey agar. The resistance of the isolates was determined using the disk diffusion method.
\end{abstract}

Results: Of 15 processed samples of birds from each hatchery, all samples from hatcheries B, D, and E, 10 (66.7\%) and 14 (93.3\%) samples from hatcheries A and C, respectively, yielded pure cultures of Escherichia coli. Klebsiella was also isolated from $1(7.1 \%)$ of the 14 and $2(13.2 \%)$ of the 15 growth-positive samples from hatcheries $\mathrm{C}$ and $\mathrm{D}$, respectively. The range of resistance among $E$. coli isolates was tetracycline (86.7-100\%), ampicillin (80-100\%), gentamicin (60-85.7\%), sulfamethoxazole-trimethoprim (46.7-92.9\%), enrofloxacin (40-100\%), ciprofloxacin (26.7-86.7\%), streptomycin (10-80\%), cefotaxime (26.7-73.3\%), amoxicillin-clavulanic acid (13.3-60\%), and ceftazidime (6.7-40\%). Klebsiella and all E. coli isolate from chicks of hatcheries B, C, and E, 80 and $93.3 \%$ of those from chicks of hatcheries A and D, respectively, exhibited resistance to three or more classes of antibacterial agents.

Conclusion: Commercial day-old broiler chicks in Nigeria are colonized by multidrug-resistant coliforms (E. coli and Klebsiella) and are potential reservoirs and disseminators of these organisms.

Keywords: avian, coliforms, day-old chicks, Enterobacteriaceae, multidrug resistant.

\section{Introduction}

The burgeoning Nigerian population (currently estimated to be 180 million with $3 \%$ annual growth rate) has resulted in unprecedented demand for animal food, of which the supply is far below the demand [1]. The poultry industry is the key livestock sector providing about $25 \%$ of animal protein and huge employment opportunities for Nigerians, with the sector increasing from 150,700 million chickens in 2005 to 192,313 million in 2010 [1-4]. In 2011, Nigeria ranked $19^{\text {th }}$ top hen egg producer in the world and top hen egg producer in Africa with total hen egg production of 636,000 metric tonnes valued at $\$ 527.49$ million $[1,5]$. In Nigeria, day-old chicks (DOCs) are sourced mostly from hatcheries in the Southwestern region of the

Copyright: Okorafor, et al. Open Access. This article is distributed under the terms of the Creative Commons Attribution 4.0 International License (http://creativecommons.org/licenses/ by/4.0/), which permits unrestricted use, distribution, and reproduction in any medium, provided you give appropriate credit to the original author(s) and the source, provide a link to the Creative Commons license, and indicate if changes were made. The Creative Commons Public Domain Dedication waiver (http:// creativecommons.org/publicdomain/zero/1.0/) applies to the data made available in this article, unless otherwise stated. country [1]. Colonization of the gut by microbiota in young animals, including broiler chicks, occurs simultaneously with the development of the gut tissues [6,7]. Members of the family Enterobacteriaceae are among the microbiota colonizing the intestinal tract of chicks, protecting against pathogenic strains through competitive exclusion [7-11]. The hot humid tropical climate of Nigeria favors the growth of enterobacteria [1]. Apart from Newcastle disease and Avian influenza and perhaps global economic crises and inadequate credit, enterobacterial diseases such as colibacillosis and salmonellosis constitute the major constraint to poultry production in Nigeria $[1,12,13]$. These diseases are responsible for heavy morbidity and mortalities, especially at early stages of broiler chicks' life [14]. Poultry farmers in Nigeria add massive amounts of different types/classes of antibacterials, including the critically important ones, in feed and/or drinking water to prevent these diseases $[15,16]$. Most times, this prevention strategy fails and the birds come down with the diseases [15]. Most antibacterials used in treating diseases in poultry are now ineffective; thus, cases of therapeutic failure and increased mortality are often 
reported in Nigeria $[4,15]$. This suggests that multidrug-resistant (MDR) bacteria might be offenders in most of these cases.

With the increasing reports of MDR enterobacteria in poultry and poultry meat worldwide, and the impact of these organisms on public health, there is increased interest in the origin of these organisms in the poultry production chain $[9,11,17]$. There is increasing number of reports on isolation of MDR enterobacteria from broilers in Nigeria $[18,19]$. Studies have shown that MDR enterobacteria could be pseudovertically transmitted through contaminated eggshell to DOCs and/or transovarianly from parent stock to hatchery $[9,11,20,21]$. Reports also showed that vertical transmission of MDR enterobacteria from parent stock to chicks from the top of production pyramid resulted in introduction and spread of resistance genes in poultry $[22,23]$. Parent stocks of commercial day-old broiler chicks in Nigeria are often sourced from overseas [1] and there are evidences that antibacterial agents, including critically important ones (such as extended-spectrum cephalosporins), are often injected in ovo in these countries [24,25]. In Nigeria, newly hatched chicks are vaccinated (usually with viral vaccines) in hatcheries and transported to production farms; they are considered "day old" until placed in production farms which are putative sources of resistant bacteria [26]. Assessing the potential of commercial day-old broiler chicks as reservoirs of resistant enterobacteria is crucial for devising strategies for improving biosecurity in hatcheries and parent stock farms, and for sourcing of parent stocks and DOCs. Determination of the antibiogram of enterobacteria colonizing healthy DOCs is important for adequate empiric treatment in these birds [14].

The spread of MDR enterobacteria in poultry industry is a matter of serious concern due to their zoonotic importance $[11,14,27]$. This is particularly important in Nigeria where poultry farming systems are not organized, and antibacterials are used to counterbalance poor hygiene and inadequate biosecurity [1]. The presence of MDR enterobacteria in dayold broiler chicks constitutes a threat to public health because these organisms could be acquired through direct and indirect contact with carrier birds, and/or consumption of undercooked poultry meat and associated products from the future broilers $[11,14,21]$. This is of great concern in Nigeria where there is no pre-slaughter assessment of antibacterial usage in broilers and some butchers consume raw chicken meat. Farmers and veterinarians are at greater risk of acquiring resistant organisms from DOCs due to frequent direct contact with these birds [14]. Passage of these organisms into the food chain/water bodies through poultry manure used in fertilizing farmlands is also of concern. This is because acquisition of these resistant organisms by humans and animals jeopardizes antimicrobial therapy in infected/carrier individuals [28]. Colonized individuals potentially serve as reservoirs and disseminators of these organisms to the environment and the public $[11,14]$. Thus, there is a need to assess antibacterial resistance profile of enterobacteria harbored by DOCs in Nigeria. Such information will be useful in devising strategy for controlling the development of antibiotic resistance in the poultry industry in Nigeria. Studies [9,10,14,29-34] in some countries showed that healthy and diseased DOCs are potential reservoirs of MDR enterobacteria. In Nigeria, a study [35] isolated antibacterial-resistant enterobacteria from dead-in-shell embryos while another [15] recovered resistant enterobacteria in clinical samples of DOCs. No study has assessed the potential of apparently healthy commercial day-old broiler chicks in Nigeria as reservoirs of MDR enterobacteria, whereas these birds are important source of the introduction of MDR organisms in broiler production chain. Surveillance of resistance in commensal enterobacteria is important because they are potential reservoirs of resistance genes and more ubiquitous than pathogens [14].

The objective of this study was to isolate enterobacteria colonizing commercial day-old broiler chicks and to determine the antibacterial and multidrug resistance profiles of the isolates.

\section{Materials and Methods}

\section{Ethical approval}

Permission to conduct the study and ethical clearance was obtained from the Medical Research Ethics Committee of the University of Nigeria, Nsukka. All the birds in this study were handled humanely in accordance with the Helsinki Declaration [36].

\section{Sampling}

This cross-sectional study was conducted between January and August 2016, in Nsukka, Southeast Nigeria. Five hatcheries (identified as A, B, C, D and E) in Southwest Nigeria, mostly patronized by DOCs distributors and farmers in the Southeastern region of the country were purposively selected for this study. Immediately on arrival, after overnight transport, five birds were randomly selected from every batch of 100 DOCs sourced from each of the hatcheries. The sampling was done 3 times at 2-week interval to increase the chances of recovering resistant organisms if there is contamination over time. A total of 15 birds were sampled per hatchery. Selected chicks were placed in a new carton obtained from the respective hatchery and transported to the Veterinary Microbiology Laboratory, University of Nigeria, Nsukka, within $5 \mathrm{~min}$. Non-duplicate cloacal swabs were collected from each of the bird using a sterile swab stick.

\section{Isolation and generic identification of enterobacteria from day-old broiler chicks}

The swabs were cultured directly on MacConkey agar and incubated at $37^{\circ} \mathrm{C}$ for $24 \mathrm{~h}$ in ambient air. Morphotypes were described and recorded appropriately. For mixed cultures, isolates were purified 
by subculturing on MacConkey agar and incubated at $37^{\circ} \mathrm{C}$ for $24 \mathrm{~h}$. Phenotypic characterization of the isolates to generic level was done by subjecting them to various tests such as Gram-staining, citrate, indole, methyl red, ornithine decarboxylase, triple sugar iron agar test, and subculturing on eosin methylene blue agar following standard procedures [37].

Determination of antibiogram of generic enterobacterial isolates from day-old broiler chicks

Antibacterial resistance/susceptibility profiles of the enterobacterial isolates were determined by the disk diffusion method using 10 antibacterial agents belonging to six classes: Fluoroquinolones - ciprofloxacin (CIP; $5 \mu \mathrm{g}$ ) and enrofloxacin (ENR; $5 \mu \mathrm{g}$ ), folate pathway antagonists - sulfamethoxazole-trimethoprim (SXT; $25 \mu \mathrm{g}$ ), $\beta$-lactams/cephalosporins ampicillin (AMP; $10 \mu \mathrm{g}$ ), ceftazidime (CTZ; $30 \mu \mathrm{g}$ ), and cefotaxime (CTX; $30 \mu \mathrm{g}), \beta$-lactam combination agents - amoxicillin-clavulanic acid (AMC; 20/10 $\mu \mathrm{g}$ ), tetracycline (TET; $30 \mu \mathrm{g}$ ), aminoglycosides - gentamicin (GEN; $10 \mu \mathrm{g})$ and streptomycin (STR; $10 \mu \mathrm{g})$. Escherichia coli American Type Culture Collection ${ }^{\circledR}$ (ATCC) 25922 and Klebsiella pneumoniae ATCC 700603 were used as reference strains. Results of the antibacterial resistance/susceptibility testing were interpreted according to the Clinical and Laboratory Standards Institute [38] guidelines for Enterobacteriaceae. Intermediately susceptible isolates were classified as resistant in this study. An isolate resistant to agents in three or more classes/categories was considered MDR [39].

\section{Statistical analysis}

The frequencies of the occurrence of enterobacterial genera and resistance of the isolates from pooled samples of birds from each hatchery were entered into Microsoft Excel version 2010 (Microsoft Corporation, Redmond, USA) and their percentages calculated. The median value of resistance to the antibacterial agents was determined using Microsoft Excel.

\section{Results}

\section{Isolation rate of generic enterobacteria from day-old} broiler chicks

Of 15 processed samples from each hatchery, all from hatcheries B, D, and E, $10(66.7 \%)$ and $14(93.3 \%)$ from hatcheries A and C, yielded only pure cultures of E. coli. Of the 14 and 15 positive samples from hatcheries C and D, 1 (7.1\%) and 2 (13.3\%) also yielded Klebsiella, respectively.

\section{Antibiogram of generic enterobacterial isolates from day-old broiler chicks}

Antibacterial resistance profiles of $E$. coli isolates from various hatcheries are presented in Table- 1 . Of the 10 E. coli isolates from chicks of hatchery A, $90 \%$ were resistant to TET and AMP, $10 \%$ to STR, $60 \%$ to GEN, AMC, CTX, and ENR, and $30 \%$ to CTZ and $40 \%$ to CIP. Of the $15 \mathrm{E}$. coli isolates from chicks of hatchery B, all were resistant to TET and AMP, $46.7 \%$ to SXT, $40 \%$ to STR, $60 \%$ to GEN, $40 \%$ to AMC,
$6.7 \%$ to CTZ, $26.7 \%$ to CTX and CIP, and $66.7 \%$ to ENR. Among the $14 \mathrm{E}$. coli isolates from chicks of hatchery $\mathrm{C}$, all were resistant to AMP and ENR, 92.9\% to SXT and TET, $28.6 \%$ to STR and CTZ, $85.7 \%$ to GEN and CIP, $21.4 \%$ to AMC, and $57.1 \%$ to CTX. Among the $15 \mathrm{E}$. coli isolates from chicks of hatchery D, 60\% were resistant to SXT and GEN, $86.7 \%$ to TET, $26.7 \%$ to STR, $80 \%$ to AMP, $20 \%$ to AMC, $6.7 \%$ to CTZ, and $40 \%$ to CTX, ENR, and CIP. Of the 15 E. coli isolates from chicks of hatchery E, all were resistant to TET and AMP, $86.7 \%$ to SXT and CIP, $80 \%$ to STR and GEN, $13.3 \%$ to AMC, $40 \%$ to CTZ, $73.3 \%$ to CTX, and $93.3 \%$ to ENR. The range of resistance among $E$. coli isolates from the hatcheries was TET $(86.7-100 \%$, median $=92.9 \%)$, AMP $(80-100 \%$, median $=100 \%)$, GEN $(60-85.7 \%$, median $=85.7 \%)$, SXT $(46.7-92.9 \%$, median $=92.9 \%)$, ENR $(40-100 \%$, median $=100 \%)$, CIP $(26.7-86.7 \%$, median $=85.7 \%)$, STR $(10-80 \%$, median $=28.6 \%)$, CTX $(26.7-73.3 \%$, median $=57.1 \%)$, AMC $(13.3-60 \%$, median $=21.4 \%)$, and CTZ $(6.7-40 \%$, median $=28.6 \%)$.

All E. coli isolates from chicks of hatcheries B, C, and E, $80 \%$ and $93.3 \%$ of those from hatcheries A and $\mathrm{D}$, respectively, exhibited resistance to at least a drug in three or more classes of antibacterial agents and thus MDR (Table-2). Both Klebsiella isolates from chicks of hatchery D were resistant to CTX, TET, and SXT (that is, three classes of antibacterial agents) while $1(50 \%)$ was resistant to AMC, GEN, AMP, CIP, ENR, and CTZ (that is, four classes of antibacterial agents). Klebsiella isolate from a chick of hatchery $\mathrm{C}$ was susceptible to only STR but exhibited resistance to all the six classes of antibacterial agents tested.

\section{Discussion}

The fact that only E. coli was isolated from all the samples from three hatcheries $(\mathrm{B}, \mathrm{D}$, and $\mathrm{E})$ and most of those from the remaining two hatcheries (A and C) in this study suggested that E. coli may be the dominant enterobacteria colonizing commercial dayold broiler chicks in Nigeria. Less frequent isolation of Klebsiella in this study suggested that it may not be a common enterobacteria genus colonizing commercial day-old broiler chicks in Nigeria. Members of the family Enterobacteriaceae are known to colonize DOCs even before they have contact with the environment $[7,11]$. Elsewhere, investigators isolated E. coli $[29,30-32,40]$ and Klebsiella [29,34] from samples of healthy DOCs. In the presented experiment, direct plating was employed in culturing the samples; this may account for why other enterobacteria genera such as Salmonella which requires pre-enrichment/ enrichment could not be isolated. The previous studies [31,41] elsewhere reported isolation of Salmonella from samples of DOCs, but Nazer et al. [29] did not isolate Salmonella from 120 healthy day-old broiler chicks despite pre-enrichment and enrichment processes. Therefore, it is also possible that the absence of Salmonella in this present study may be because 
Table-1: Antibiogram of Escherichia coli isolates from day-old broiler chicks from different hatcheries.

\begin{tabular}{lccccc}
\hline Antibacterial agent (concentration) & \multicolumn{4}{c}{ Percentage of resistant isolates per hatchery } \\
\cline { 2 - 6 } & A (n=10) & B (n=15) & C (n=14) & D (n=15) & E (n=15) \\
\hline Sulfamethoxazole-trimethoprim $(25 \mu \mathrm{g})$ & 0 & 46.7 & 92.9 & 60 & 86.7 \\
Tetracycline $(30 \mu \mathrm{g})$ & 90 & 100 & 92.9 & 86.7 & 100 \\
Ampicillin $(10 \mu \mathrm{g})$ & 90 & 100 & 100 & 80 & 100 \\
Ceftazidime $(30 \mu \mathrm{g})$ & 30 & 60.7 & 28.6 & 6.7 & 40 \\
Cefotaxime $(30 \mu \mathrm{g})$ & 60 & 26.7 & 57.1 & 40 & 73.3 \\
Amoxicillin-clavulanic acid $(20 / 10 \mu \mathrm{g})$ & 60 & 40 & 21.4 & 20 & 13.3 \\
Streptomycin $(10 \mu \mathrm{g})$ & 10 & 40 & 28.6 & 26.7 & 80 \\
Gentamicin $(10 \mu \mathrm{g})$ & 60 & 60 & 85.7 & 60 & 80 \\
Enrofloxacin $(5 \mu \mathrm{g})$ & 60 & 66.7 & 100 & 40 & 93.3 \\
Ciprofloxacin $(5 \mu \mathrm{g})$ & 40 & 26.7 & 85.7 & 40 & 86.7 \\
\hline
\end{tabular}

Table-2: Resistance of Escherichia coli isolates from day-old broiler chicks from different hatcheries to antibacterial classes.

\begin{tabular}{|c|c|c|c|c|c|}
\hline \multirow{2}{*}{$\begin{array}{l}\text { Number of } \\
\text { antibacterial class }\end{array}$} & \multicolumn{5}{|c|}{ Percentage of resistant isolates per hatchery } \\
\hline & $A(n=10)$ & $B(n=15)$ & $C(n=14)$ & $D(n=15)$ & $E(n=15)$ \\
\hline 1 & 0 & 0 & 0 & 6.7 & 0 \\
\hline 2 & 20 & 0 & 0 & 0 & 0 \\
\hline$\geq 3$ & 80 & 100 & 100 & 93.3 & 100 \\
\hline
\end{tabular}

there was no contamination of the eggs during formation in the oviduct, during or after oviposition [42]. Chicks are hatched with sterile intestinal tract, but the microbial populations of DOCs (chicks that have neither eaten nor placed in production farm) vary due to differences in bacteria ingestion from hatching debris, environment, and production facility [26].

The moderate to high rate of resistance exhibited by isolates in this study suggested selection probably due to acquisition of genes encoding resistance to the drugs. The AMP resistance (80-100\%) among E. coli isolates in this study is higher than 70\% AMP resistance among E. coli isolates from DOCs in Spain [14]. Das et al. [32] reported $82.98 \%$ AMP resistance among $E$. coli isolates from DOCs in Bangladesh, this result is within the AMP resistance range (80-100\%) but lower than the median value (100\%) in this study. JiménezBelenguer et al. [14] reported 30\% TET resistance, a finding that is lesser than that $(86.7-100 \%)$ of this study while Das et al. [32] recorded 100\% TET resistance which is comparable to the result $(80-100 \%$, median $=100 \%$ ) of this study. Zibandeh et al. [43] recorded 67\% TET resistance among E. coli isolates from 1-day-old chicks in Iran; this finding is lower than the result $(86.7-100 \%)$ of this study. JiménezBelenguer et al. [14] reported $<10 \%$ STR and GEN resistances, whereas Das et al. [32] did not observe GEN resistance; these findings contrasted the results of this study. Das et al. [32] reported 14.89\% ENR resistance which is lower than the finding (40-100\%) of this study. Nazer et al. [29] and Abdi-Hachesoo et al. [30] observed 48.97 and 41.9\% ENR resistance among $E$. coli isolates from day-old chicks in Iran, respectively; these results are in the range (40-100\%) of ENR resistance but lower than the median value $(100 \%)$ in this study. Abdi-Hachesoo et al. [30] also reported $36.2 \%$ CIP resistance which is in the range (26.7-86.7\%) of CIP resistance but lower than the median value (85.7\%) in this study, whereas JiménezBelenguer et al. [14] and Das et al. [32] reported 16 and $25.53 \%$ CIP resistance, respectively, which are lower than the result of this study. Biswas et al. [31] reported that E. coli isolates from day-old broiler chicks in Bangladesh exhibited high susceptibility to GEN, moderate susceptibility to CIP, and less susceptibility to TET. Variation in resistance among isolates in these studies could be related to the differences in susceptibility testing method(s) employed, poultry management, level of contamination of hatcheries from where the birds were sourced, and usage of antibacterials in the study areas. In this study, the medical history of the parent stock of the sampled chicks and antibacterial usage in the various hatcheries were not traced.

The fact that $80-100 \%$ of E. coli and Klebsiella isolates in this study were MDR (exhibited resistance to agents in three or more classes [39]) indicated that the birds are potential reservoirs of these organisms $[14,44]$. This finding suggested that the isolates could have acquired genes encoding resistance to the various antibacterials probably due to selection pressure [14,19]. This finding of resistance to many classes of antibacterial suggested that these agents have been tremendously abused in Nigerian poultry industry due to uncontrolled use of antimicrobials in the country [19].

The finding of multidrug resistance in this study calls for serious concern due to limited option for empiric therapy if the birds become infected with these organisms $[12,14,30]$. This could result in large-scale mortality and resorting to the use of last-line antibacterials such as colistin for the prevention and treatment 
of poultry diseases in Nigeria. Unfortunately, there is now a described plasmid-mediated colistin resistance (with different genes that have been described to date) [45]. Moreover, these organisms pose a serious threat to the health of individuals that acquire them on making direct contact (such as poultry farmers, veterinarians, and consumers of meat and associated products derived from these future broilers) with the birds. These organisms could be transmitted to the public through the primary contacts and/or poultry manure used in fertilizing farmlands and subsequently contaminating the food chain $[11,14,30]$. In this study, usage of antibacterials in the parent stock and hatcheries were not traced. However, possible sources of MDR organisms in the sampled birds include: Contaminated parent stock, egg surfaces, hatcheries/hatchery workers, and/or during transportation [14,20,25,34].

\section{Conclusion}

$E$. coli is the dominant genus of enterobacteria colonizing commercial day-old broiler chicks in Nigeria. High percentages $(80-100 \%)$ of commercial day-old broiler chicks in Nigeria are colonized by MDR enterobacteria. Thus, these birds are potential reservoirs and disseminators of these organisms and genes encoding resistance to different classes of antibacterial agents. This has a tremendous impact on the food chain and epidemiology of antibacterial resistance. Therefore, in Nigeria, the acquisition of broiler parent stock should be done with caution and attention should be paid on the use of antibacterials in the poultry industry. However, further studies to determine the resistance genes harbored by the isolates are recommended.

\section{Authors' Contributions}

ONO, GNA, and RIU conceptualized the study. ONO, MUA, and EON collected and processed the samples. All authors participated in draft and revision of the manuscript. All authors read and approved the final manuscript.

\section{Acknowledgments}

The authors are grateful to Prof. Kennedy Chah for providing technical assistance in this study. This study did not receive any external funding.

\section{Competing Interests}

The authors declare that they have no competing interests.

\section{Publisher's Note}

Veterinary World remains neutral with regard to jurisdictional claims in published institutional affiliation.

\section{References}

1. Fagbamila, S.I.O., Barco, L., Mancin, M., Kwaga, J., Ngulukun, S.S., Zavagnin, P., Lettini, A.A., Lorenzetto, M., Abdu, P.A., Kabir, J., Umoh, J., Ricci, A. and
Muhammad, M. (2017) Salmonella serovars and their distribution in Nigerian commercial chicken layer farms. PLoS One, 12(3): e0173097.

2. Food and Agricultural Organization. (2008) Assessment of the Nigerian Poultry Market Chain to Improve Biosecurity. Available from: http://www.fao.org/3/a-ak778e.pdf. Last accessed on 15-01-2019.

3. Agada, G.O.A., Abdullahi, I.O., Odugbo, A.M., Chollom, S.C., Kumbish, P.R. and Okwori, A.E.J. (2014) Prevalence and antibiotic resistance profile of Salmonella isolates from commercial poultry and poultry farm handlers in Jos, Plateau State, Nigeria. Br. Microbiol. Res. J., 4(4): 462-479.

4. Balami, A.G., Ndahi, J.J., Zaifada, A.U., Mustapha, M., Jarafu, D.J., Asogwa, N.T. and Hajara, S. (2014) A retrospective study of poultry diseases diagnosed in Maiduguri, North-East, Nigeria. Poult. Fish. Wildl. Sci., 2: 113.

5. Food and Agricultural Organization. (2015) Data Source 2015: FAOSTAT FAO Statistics Division 2015. Food and Agricultural Organization, Rome.

6. Chung, H., Pamp, S.J., Hill, J.A., Surana, N.K., Edelman, S.M., Troy, E.B., Reading, N.C., Villablanca, E.J., Wang, S., Mora, J.R., Umesaki, Y., Mathis, D., Benoist, C., Relman, D.A. and Kasper, D.L. (2012) Gut immune maturation depends on colonization with a host-specific microbiota. Cell, 149(7): 1578-1593.

7. Schokker, D., Jansman, A.J.M., Veninga, G., de Bruin, N., Vastenhouw, S.A., de Bree, F.M., Bossers, A., Rebel, J.M.J. and Smits, M.A. (2017) Perturbation of microbiota in one-day-old broiler chickens with antibiotic for 24 hours negatively affects intestinal immune development. $B M C$ Genom., 18: 241.

8. Ranjitkar, S., Lawley, B., Tannock, G. and Engberg, R.M. (2016) Bacterial succession in the broiler gastrointestinal tract. Appl. Environ. Microbiol., 82(8): 2399-2410.

9. Projahn, M., Daehre, K., Roesler, U. and Friese, A. (2017a) Extended-spectrum beta-lactamase and plasmid-encoded cephamycins-producing enterobacteria in broiler hatchery as a potential mode of pseudovertical transmission. Appl. Environ. Microbiol., 83(1): e02364.

10. Projahn, M., Daehre, K., Semmler, T., Guenther, S., Roesler, U. and Friese, A. (2017b) Environmental adaptation and vertical dissemination of ESBL-/pAmpC-producing Escherichia coli in an integrated broiler production chain in the absence of antibiotic treatment. Microb. Biotechnol., 11(6): 1017-1026.

11. Osman, K.M., Kappell, A.D., Elhadidy, M., ElMougy F., Abd El-Ghany, W.A., Orabi, A., Mubarak, A.S., Dawoud, T.M., Hemeg, H.A, Moussa, I.M.I, Hessain, A.M. and Yousef, H.M.Y. (2018) Poultry hatcheries as potential reservoirs for antimicrobial resistant Escherichia coli: A risk to public health and food safety. Sci. Rep., 8(1): 5859.

12. Kabir, S.M.L. (2010) Avian colibacillosis and salmonellosis: A closer look at epidemiology, pathogenesis, diagnosis, control and public health concerns. Int. J. Environ. Res. Public. Health, 7(1): 89-114.

13. Mamman, P.H., Kazeem, H.M., Raji, M.A., Nok, A.J. and Kwaga, J.K.P. (2014) Isolation and characterization of Salmonella Gallinarum from outbreaks of fowl typhoid in Kaduna State, Nigeria. J. Public. Health Epidemiol., 3(10): 82-88.

14. Jiménez-Belenguer, A., Doménech, E., Villagrá, A., Fenollar, A. and Ferrús, M.A. (2016) Antimicrobial resistance of Escherichia coli isolated in newly hatched chickens and effect of amoxicillin treatment during their growth. Avian Pathol., 45(4): 501-507.

15. Anyanwu, A.L., Fasina, F.O., Ajayi, O.T., Rapu, I. and Fasina, M.M. (2010) Antibiotic-resistant Salmonella and Escherichia coli isolated from day-old chicks, Vom, Nigeria. Afr. J. Clin. Exp. Microbiol., 11(1): 129-136.

16. Ojo, E.O., Eniola, F., Majsan, A.A. and Dipeolu, M.A. (2015) Antimicrobials in animal production: Usage and 
practices among livestock farmers in Oyo and Kaduna States of Nigeria. Trop. Anim. Health Prod., 48(1): 189-197.

17. Mezhoud H, Chantziaras I, Iguer-Ouada M, Moula N, Garmyn A, Martel A, Touati A, Smet A, Haesebrouck F. and Boyen, F. (2016) Presence of antimicrobial resistance in coliform bacteria from hatching broiler eggs with emphasis on ESBL/AmpC-producing bacteria. Avian Pathol., 45(4): 493-500

18. Ojo, O.E., Schwarz, S. and Michael, G.B. (2016) Detection and characterization of extended-spectrum $\beta$-lactamase-producing Escherichia coli from chicken production chains in Nigeria. Vet. Microbiol., 15(194): 62-68.

19. Alonso, C., Zarazaga, M., Ben Sallem, R., Jouini, A., Ben Slama, K. and Torres, C. (2017) Antibiotic resistance in Escherichia coli in animal husbandry: The African perspective. Lett. Appl. Microbiol., 64(5): 318-334.

20. Dierikx, C.M., Van der Goot, J.A., Smith, H.E., Kant, A and Mevius, D.J. (2013) Presence of ESBL/AmpCproducing Escherichia coli in the broiler production pyramid: A descriptive study. PLoS One, 8(11): e79005.

21. Daehre, K., Projahn, M., Semmler, T., Roesler, U. and Friese, A. (2017) Extended-spectrum beta-lactamase-/ AmpC beta-lactamase-producing Enterobacteriaceae in broiler farms: Transmission dynamics at farm level. Microb. Drug Resist., 24(4): 511-518.

22. Laube, H., Friese, A., von Salviati, C., Guerra, B., Käsbohrer, A., Kreienbrock, L. and Roesler, U. (2013) Longitudinal monitoring of extended-spectrum-beta-lactamase/AmpC-producing Escherichia coli at German broiler chicken fattening farms. Appl. Environ. Microbiol., 79(16): 4815-4820.

23. Borjesson, S., Ny, S., Egervarn, M., Bergstrom, J., Rosengren, A., Englund, S., Lofmark, S. and Byfors, S. (2016) Limited dissemination of extended-spectrum beta-lactamase- and plasmid-encoded AmpC-producing Escherichia coli from food and farm animals, Sweden. Emerg. Infect. Dis., 22(4): 634-640.

24. McReynolds, J.L., Caldwell, D.Y., Barnhart, E.T., Deloach, J.R., McElroy, A.P., Moore, R.W., Hargis, B.M. and Caldwell, D.J. (2000) The effect of in ovo or day-ofhatch subcutaneous antibiotic administration on competitive exclusion culture (PREEMPT) establishment in neonatal chickens. Poult. Sci. J., 79(11): 1524-1530.

25. Baron, S., Jouy, E., Larvor, E., Eono, F., Bougeard, S. and Kempf, I. (2014) Impact of third-generation-cephalosporin administration in hatcheries on fecal Escherichia coli antimicrobial resistance in broilers and layers. Antimicrob. Agents Chemother., 58(9): 5428-5434.

26. Binek, M., Borzemska, W., Pisarski, R., Taszczak, B., Kosowska, G., Malec, H. and Karpinska, E. (2000) Evaluation of the efficacy of feed providing on development of gastrointestinal microflora of newly hatched broiler chickens. Eur. Poult. Sci., 64(4): 147-151.

27. Nhung, N.T., Chansiripornchai, N. and Carrique-Mas, J.J. (2017) Antimicrobial resistance in bacterial poultry pathogens: A review. Front. Microbiol., 4: 126.

28. Ljubojević, D., Puvaca, N., Pelić, M., Todorović, D., Pajić, M., Milanov. D. and Velhner, M. (2016) Epidemiological significance of poultry litter for spreading the antibiotic-resistant strains of Escherichia coli. Worlds Poult. Sci. J., 72(3): 485-494.

29. Nazer, A.H.K., Dandras, H. and Eskandari, S. (2006) Aerobic bacteria isolated from eggs and day-old chicks and their antibacterial resistance in Shiraz, Iran. Iran. J. Vet. Res., 7(2): 20-30.

30. Abdi-Hachesoo, B., Asasi, K. and Sharifiyazdi, H. (2013) Rapid detection of Escherichia coli gyrA and parC mutants in one-day-old broiler chicks in Iran. Vet. Ital., 49(3): 291-297.

31. Biswas, S., Akter, M.R., Chaki, A.R., Ikbal, S.M.,
Matin, M.A. and Alam, M.S. (2015) Antimicrobial sensitivity of bacterial pathogens isolated from day-old broiler. Int . J. Nat. Soc. Sci., 2(5): 56-63.

32. Das, A., Sen, A., Dhar, P.K., Nath, S.K., Ghosh, P. and Saifuddin, A.K.M. (2017) Isolation of Escherichia coli from the liver and yolk sac of day-old chicks with their antibiogram. Br. J. Biomed. Multidiscip. Res., 1(1): 19-25.

33. Attia, A.M., Hassan, M.N. and Elwan, S.E. (2017) Molecular characterization of $\beta$-lactamase genes in antibiotic-resistant bacteria. Zagazig Vet. J., 45(1): 55-63.

34. Yossapol, M., Sugiyama, M. and Asai, T. (2017) The occurrence of CTX-M-25-producing Enterobacteriaceae in day-old broiler chicks in Japan. J. Vet. Med. Sci., 79(10): 1644-1647.

35. Raji, M., Adekeye, J., Kwaga, J., Bale, J. and Henton, M. (2007) Serovars and biochemical characterization of Escherichia coli isolated from colibacillosis cases and dead-in-shell embryos in poultry in Zaria Nigeria. Vet. Arhiv, 77(6): 495-505.

36. World Medical Association. (2013) World medical association declaration of Helsinki: Ethical principles for medical research involving human subjects. J. Am. Med. Assoc., 310(20): 2191-2194.

37. Cheesebrough, M. (2000) District Laboratory Practice in Tropical Countries Part 2. Cambridge University Press, Cambridge. p63-70.

38. Clinical and Laboratory Standards Institute. (2018) Performance Standards for Antimicrobial Susceptibility Testing. $28^{\text {th }}$ ed. CLSI Supplement M100, Wayne, PA.

39. Magiorakos, A.P., Srinivasan, A., Carey, R.B., Carmeli, Y., Falagas, M.E., Giske, C.G., Harbarth, S., Hindler, J.F., Kahlmeter, G., Olsson-liljequist, B., Paterson, D.L., Rice, L.B., Stelling, J., Struelens, M.J., Vatopoulos, A., Weber, J.T. and Monnet, D.L. (2012) Multidrug-resistant, extensively drug-resistant and pandrug-resistant bacteria: An international expert proposal for interim standard definitions for acquired resistance. Clin. Microbiol. Infect., 18(3): 268-281

40. Rashid, M.A., Chowdhury, K.A., Mahmud, S.M.S., Chowdhury, T., Bhuyan, A.A.M., Nahar, Z. and Paul, S.K. (2013) Escherichia coli form day-old chicks of a selected breeder farm in Bangladesh. Vet Scan, 7(2): 36-45.

41. de Albuquerque, H.A., Maciel, W.C., Lopes, E.D.S., Teixeira, R.S.C., Salles, R.P.R., Machado, D.R., Bezerra, W.G.A, Vasconcelos, R.H., Mendonça, S.V. and Carbó, C.B. (2014) Presence of Salmonella spp. in oneday-old chicks from hatcheries in the metropolitan region of Fortaleza, Brazil. Acta Sci. Vet., 42: 1222.

42. Moosavy, M., Esmaeili, S., Amiri, F.B., Mostafavi, E. and Salehi, T.Z. (2015) Detection of Salmonella spp. in commercial eggs in Iran. Iran. J. Microbiol., 7(1): 50-54.

43. Zibandeh, S., Sharifiyazdi, H., Asasi, K. and AbdiHachesoo, B. (2016) Investigation of tetracycline resistance genes in Escherichia coli isolates from broiler chickens during a rearing period in Iran. Vet. Arhiv, 86(4): 565-572.

44. Exner, M., Bhattacharya, S., Christiansen, B., Gebel, J., Goroncy-Bermes, P., Hartemann, P., Heeg, P., Ilschner, C., Kramer, A., Larson, E., Merkens, W., Mielke, M., Oltmanns, P., Ross, B., Rotter, M., Schmithausen, R.M., Sonntag, H. and Trautmann, M. (2017) Antibiotic resistance: What is so special about multidrug-resistant Gramnegative bacteria? GMS Hyg. Infect. Cont., 12: Doc05.

45. Wang, X., Wang, Y., Zhou Y., Li, J., Yin, W., Wang, S., Zhang, S., Shen, J., Shen, Z. and Wang, Y. (2018) Emergence of a novel mobile colistin resistance gene, $m c r-8$, in NDMproducing Klebsiella pneumoniae. Emerg. Microbes Infect., $7(1): 122$ 